\title{
Le mort circoncis. Le culte des crânes dans les populations de la Haute Bénoué (Cameroun / Nigeria)
}

The Desceased Circumcised. Skull Cults among the Peoples of the Upper Benue River Basin (Cameroon and Nigeria)

\section{Françoise Dumas-Champion}

\section{OpenEdition Journals}

Édition électronique

URL : https://journals.openedition.org/span/1114

DOI : $10.4000 /$ span. 1114

ISSN : 2268-1558

\section{Éditeur}

École pratique des hautes études. Sciences humaines

\section{Édition imprimée}

Date de publication : 1 novembre 1989

Pagination : 33-74

ISSN : 0294-7080

\section{Référence électronique}

Françoise Dumas-Champion, « Le mort circoncis. Le culte des crânes dans les populations de la Haute Bénoué (Cameroun / Nigeria) », Systèmes de pensée en Afrique noire [En ligne], 9 | 1989, mis en ligne le 15 octobre 2013, consulté le 03 août 2021. URL : http://journals.openedition.org/span/1114 ; DOI : https://doi.org/10.4000/span.1114 


\section{LE MORT CIRCONCIS}

\section{Le culte des crânes dans les populations de la Haute Bénoué (Cameroun/Nigeria)}

par

\section{Françoise Dumas-Champion}

Les pratiques d'exhumation et de conservation des crânes en Afrique ne semblent avoir retenu l'attention des ethnologues que de manière secondaire ou subordonnée. Hormis les descriptions faites par certains spécialistes du monde bantou des traitements que réservent ces populations aux crânes des chefs et des rois, les données sur le sujet sont rares et fragmentaires, particulièrement lorsqu'il s'agit des gens du commun. L'importance d'une telle pratique parmi les peuples de la Haute Bénoué ${ }^{1}$ nous a amené à considérer cette question en prenant pour principal objet de recherche la place qu'occupe le traitement du crâne dans ce qu'on pourrait appeler le processus d'ancestralisation du défunt.

1 Dans les sociétés segmentaires sans pouvoir centralisé, comme chez les Koma, Dowayo, Papé, Véré, Munựé, Mumbake, Gabin, on conserve le crâne de tous les individus. Il devient un instrument cultuel qui joue un rôle social de premier ordre. Dans les systèmes monarchiques ò̀ cette pratique est réservée au roi, le crâne fait fonction de regalia. C'est le cas des Duru, Bata, Bachama, Chamba, Daka, Kan, Yungur du Sud. A propos des Jukun, Meek écrit: "il est probable que la préservation du crâne était répandue dans toutes les communautés jukrun, mais actuellement seuls les Kona conservent les crânes de leurs chef s $^{\prime 1}$ (1931a:264).

Systèmes de pensée en Afrique Noire, 9, 1989 
Dans un premier temps, nous suivrons, chez les Koma du Cameroun $^{2}$, le déroulement des opérations rituelles qui vont de l'enterrement à l'exhumation du crâne. Les matériaux utilisés ici ne sont pas sans évoquer certaines des conceptions indonésiennes relatives à la mort qui, selon l'analyse qu'en a fait Robert Hertz (1907), établissent une série de corrélations entre la dessication du corps, l'évolution des conduites de deuil et le devenir de l'âme. Dans un second temps, nous nous proposons d'examiner, à partir de l'exemple des Dowayo, la reprise de thèmes liés à l'initiation dans les rites funéraires. Les Dowayo, qui font partie d'une aire culturelle ${ }^{3}$ où se retrouvent des traits communs aux rites de circoncision et au culte des crânes, développent une théorie que nous utiliserons comme référent pour exposer et expliciter les caractères récurrents de cet ensemble de populations. Elle nous permettra de proposer une hypothèse quant à la signification de la pratique de conservation des crânes.

2 Les données sur les Koma du Cameroun ont été recueillies au cours de deux enquêtes de terrain en 1983 et 1985. Les Koma sont divisés en quatre groupes politiquement autonomes comprenant d'est en ouest : les Goonu, les Gumbe, les Ritibe et les Grme. Ils parlent trois dialectes différents, rattachés, selon la classification de Greenberg, au groupe 4 des langues de l'Adamaoua de l'embranchement Niger-Congo. Ces quatre groupes, comme les autres populations de la Haute Benoué, ont subi l'influence culturelle des Chamba et des Jukun. Tous ces peuples ont un passé commun. D'après l'historien camerounais Eldridge Moharmadou, ils faisaient partie de la confédération Kona, l'une des composantes, avec les confédérations Mbum et Jukun, de l'empire de Kororafa du XVIleme au XVIIIeme siècle (1978:9). Les systèmes religieux de ces populations présentent des traits communs. Les Jukun, Chamba et Koma, entre autres, comprennent sous une même dénomination les esprits ancestraux et les divinités alors que les parents défunts sont désignés par un autre terme. Cette distinction terminologique tend à montrer que le lien de parenté, encore présent dans la mémoire des vivants, empêche l'accès au statut de "divinité". Soulignons la faible profondeur de mémoire généalogique de ces populations, qui ne retiennent pas le nom de leurs aïeux au-delà de trois générations du côté paternel conme du côté maternel.(Pour les Jukun, ef. Yamaguchi, 1974:5, Fardon pour les Chamba; Edwards pour les Vote, Dumas-Champion 1986 et 1987 pour les Koma).

3 Les Dowayo appartiennent à la même famille linguistique que les Roma. Tout au long de cette recherche, nous étayerons notre démonstration par des exemples pris dans d'autres populations de la Haute Benoué et l'Ouest Cameroun, comne les Bamum et les Bamileke qui conservent également les crânes d'ancêtres domestiques ou royaux. 


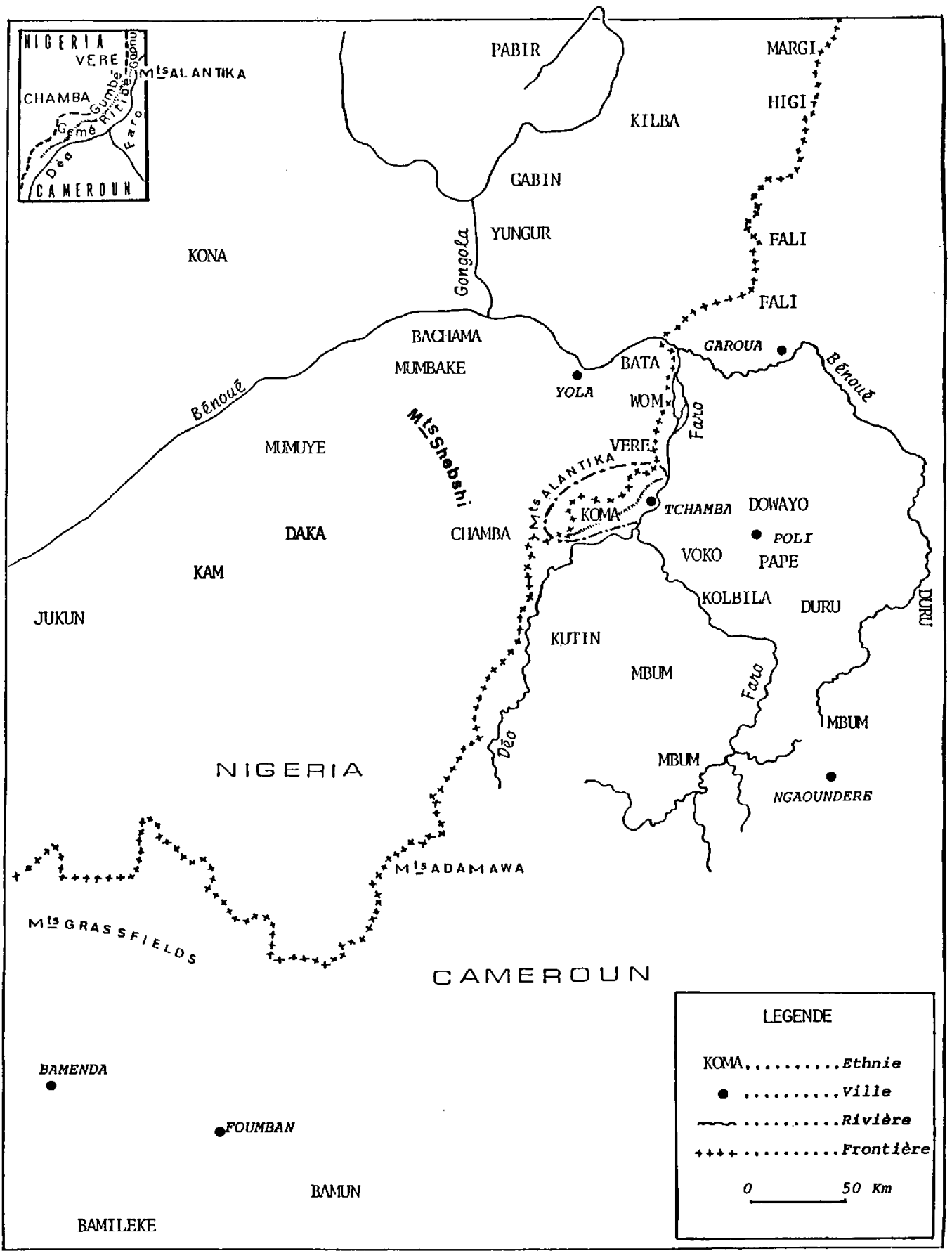

AIRE DE REPARTITION DU CULTE DES CRANES DANS LES POPULATIONS OE LA HaUte beNOUE ET DE L'OUEST CAMEROUN, 


\section{De l'enterrement à l'exhumation du crâne}

Notre propos n'est pas de présenter le détail du cycle funéraire koma mais de privilégier quelques rites significatifs concernant la position du cadavre dans la tombe, le traitement des veufs et des deuilleurs ainsi que les étapes qui mènent à l'exhumation du crâne, afin d'appréhender la corrélation entre le travail du deuil et le passage à l'ancestralité.

La préparation du cadavre vise en premier lieu à fermer tous les orifices du corps. La communication est rompue avec le défunt et l'héritier du crâne a pour charge de lui fermer les yeux. La bouche, les narines et les oreilles sont obturées avec du kapok pour éviter tout écoulement de sang ou de salive, puis la bouche est baillonnée à l'aide d'un tissu. Les Jukun, qui ferment également les orifices avec du coton et placent un baillon sur la bouche, font glisser ce dernier sur le menton au moment où le cadavre est introduit dans la niche qui lui sert de tombe ${ }^{4}$. Après le lavage et l'onction de la tête, on enroule le corps nu dans des bandes de cotonnade, à la manière d'une momie. Mais on veille à ne pas entraver le cours naturel de la séparation tête/corps qui se produira lors du pourrissement des chairs en laissant le cou libre de toute bandelette. Les Koma ne décapitent pas le cadavre, comme c'est la coutume chez les Bamun, mais favorisent la décollation de la tête en plaçant le corps verticalement dans la tombe et en le maintenant en suspension par la tête à l'aide de bois fourchus qui traversent de part en part la fosse circulaire. La décomposition des chairs provoquera la chute du reste du squelette au fond de la tombe. Seuls le crâne et la mâchoire resteront sur les branchages. Bien que la position du cadavre dans la tombe varie selon les groupes, le principe reste le même. Les Goonu sont enterrés couchés sur le dos, les bras le long du corps; la tête, orientée vers l'est, est maintenue en position verticale grâce à une sorte de tribart soutenu par des branches

4 Ce geste prend toute sa portée dans les jours qui suivent l'enterrement, lors de l'interrogatoire du défunt qui doit révéler aux vivants les causes de la mort. Celui qui prend la parole au nom du défunt donnera la réponse établie par la consultation divinatoire. 
fourchues. Ce type d'ensevelissement est, à Douga, réservé aux prêtres de Hwaye (Meek 1931a: 221). Chez les Duru, pour les défunts dont on conserve le crâne - chef, forgeron, notable ou vieillardle cadavre est placé debout dans une fosse circulaire. On laisse dépasser la tête à la surface du sol. La fosse est comblée de manière à ce que seules la tête et les mains fassent saillie. On les recouvre d'une grosse poterie dont les bords sont colmatés (Frobenius 1925/1984: 243). Nous serons plus loin en mesure d'appréhender la finalité de ce traitement particulier de la tête. Nous verrons aussi, à propos des Dowayo, que la tête est la partie du cadavre qui est toujours traitée de manière indépendante.

La tête des veuves (ou du veuf) va être l'objet de soins qui rappellent ceux qu'on prodiguera plus tard au crâne exhumé. Après l'enterrement, le forgeron, qui tient aussi le rôle de fossoyeur, va raser leur chevelure et enduire leur tête d'huile et d'ocre. Cette onction est répétée matin et soir durant les sept jours de leur réclusion. Occupées à chasser les images du mort de leurs pensées, les veuves s'aident dans ce travail sur la mémoire en se massant les tempes à l'aide d'une plante qui, dit-on, favorise l'oubli. Le devin est le fournisseur de ce végétal, un cissus quadrangularis. Le comportement mélancolique qui s'empare des deuilleurs, et pas seulement des veuves, résulte, pense-t-on, de la pénétration du défunt dans le corps de ses proches. Cette incorporation traduit en fait le malaise $\mathrm{du}$ mort concernant son nouveau statut d'errant. C'est non pas par vengeance mais par amour des siens qu'il "s'accroche" à son ancienne vie. Il est, pour les vivants, appréhendé comme une présence pathogène qu'il faut extraire du corps. C'est ce qu'indique la gestuelle qui accompagne l'onction. Il s'agit, à l'aide du pouce et de l'index, préalablement imprégnés de cissus, de masser tout d'abord les tempes du patient - on procède de la même manière pour chasser un mal de tête - puis de poursuivre le massage au niveau du sternum, considéré comme le lieu du souvenir, pour enfin glisser le long du corps jusqu'aux gros orteils, en un geste d'expulsion. Cette pratique thérapeutique est destinée à déloger le mort ancré dans l'enveloppe corporelle du deuilleur. 
L'autre temps fort du rituel, dit dog lele en gema (langue des Geme), litt: "attacher la corde", qui suit l'enterrement, consiste pour la famille du défunt à passer une ficelle autour du cou des veuves (ou du veuf). Cette corde vient rappeler au survivant les liens qui l'unissent au mort en lui interdisant tout commerce sexuel; la transgression de cet interdit entrainerait la mort de l'amant. Les Koma associent le veuvage à l'abstinence sexuelle qui en résulte, comme en témoigne le sens du terme wol yiké (ou yin wolé), litt. "l'abstinence de la mort". La sexualité d'un couple engage une énergie dont l'activité perdure par-delà la mort, et c'est cette force qui anéantirait l'amant. L'un des aspects des rites de veuvage sera de procéder au désinvestissement de cette énergie sexuelle par une période de chasteté qui sera symboliquement rompue par la rupture de la corde. Cette période varie de trois à six mois pour les femmes. Elle est généralement abrégée à deux semaines pour un homme.

Commence alors le lent travail de deuil où il va falloir, jour après jour, chasser le mort de son esprit. Si le défunt a pu être, dans un premier temps, expulsé du corps des veuves, il est encore présent parmi les vivants, présent sur les lieux mêmes de son habitation, présent à travers ses objets. Il faudra du temps pour que s'estompe son souvenir, du temps pour que "les deuilleurs consomment la mort dans leur conscience", selon les termes de Hertz (1928). A 1'altération du souvenir correspond, sous terre, une lente décomposition des chairs. Au fil des mois, les traits marquant l'individualité du mort perdent de leur netteté tant dans la mémoire des vivants que dans la réalité organique. L'errance à laquelle est voué le défunt est rapportée au temps de décomposition du cadavre. Son passage du monde des vivants vers le monde des ancêtres est tributaire du travail qui s'opère sur son enveloppe corporelle. Le squelette une fois décharné, les os s'affaisseront dans la tombe, et la tête sera séparée du corps. Les Koma qui ouvrent les sépultures pour exhumer des crânes sont très au fait de cette transformation de la forme et de la matière, qu'ils associent au changement de statut du défunt.

Le cadavre transformé en squelette, vient alors pour les vivants le temps de l'oubli : kunde disent les Grme. Le rite de levée de deuil 
qui mettra un terme à l'emprise du mort sur ses épouses rendra, dans le même temps, ses biens accessibles aux héritiers. Le fils aîné du défunt, organisateur de la cérémonie ${ }^{5}$, veille en premier lieu au brassage de la bière faite à partir du sorgho provenant de la récolte de son père. Ce breuvage qui occupe une place importante dans tous les rituels est chargé d'une haute valeur symbolique chez les Koma comme dans l'ensemble des populations de la Bénoué. Le mot "bière" intervient dans l'appellation de chaque fête, comme du reste chez les Jukun et chez les Chamba, et connote l'idée de rassemblement. Les Gumbé désignent cette séquence funéraire par le terme war vume: "la bière du cadavre", ou sul vume : "la bière de la corde" selon qu'ils privilégient ou non la rupture en jeu dans cette fin de deuil. Les Goonu emploient le terme gaéle sao: "la bière de la tombe". Les Chamba la désignent comme la "bière de la mort": weisimi (Fardon 1980: $I I, 73)^{6}$.

En signe d'ultime séparation avant la distribution de la boisson, les jarres de bière qui étaient entreposées dans l'habitation du défunt sont mises dehors ainsi que ses effets. Rien ne reviendra plus dans sa demeure. On procède au partage des jarres, selon l'ordre de séniorité. L'aîné du village, qui tient le rôle d'officiant, verse une petite quantité de bière sur le sol. On dit que c'est la part du défunt, celle qu'il attend pour partir. Chaque participant offrira au mort une semblable libation avant de consommer la bière qui est répartie convivialement entre membres de même classe d'âge, réunissant parents, amis et alliés du défunt. En conviant une dernière fois le mort à boire sa bière, on appelle à venir honorer de leur présence les esprits ancestraux qui doivent accomplir leur rôle de psychopompe. La participation des ancêtres au départ du défunt se fait toujours par la médiation de la bière, même dans le cas d'un jeune (on réduit alors considérablement la quantité de boisson en ne préparant que

5 Cette cérémonie a lieu en saison sèche, après la fête des récoltes.

"Les Véré la désignent come "la bière des cheveux", wak suuk. On se rase la tête au moment de la mort et une nouvelle fois lors de cette cérémonie (A. Edwards, documents non publiés). 
six ou sept pots, et l'on s'adresse à lui comme s'il s'agissait non pas de bière mais d'eau).

Tandis qu'on célèbre le départ du défunt, on va procéder à la libération de ses épouses et au partage de ses biens, hérités par les neveux utérins. La corde qui avait été nouée autour du cou des veuves et qui symbolise la pérennité du lien conjugal par delà la mort, est alors détachée. Tout se passe comme si ce lien s'était usé avec le temps, de même que, sous terre, le cou qui sépare la tête du reste du corps s'est rompu, libérant le défunt de son statut d'errant et les veuves de leur sujétion. La décapitation apparaît comme un modèle pour la séparation du défunt d'avec ses épouses et pour son accession à l'ancestralité. Désormais, rien ne retient plus les veuves: "Prends ta part", dit-on à chacune d'elles, "tu as vécu avec ton mari. Il t'a laissée. Prends une part de sa richesse". La veuve quittera alors la famille de son mari et se remariera ailleurs, à moins qu'elle accepte de rester avec un frère du mort.

Le rite final inaugure le nouveau statut du défunt et apporte la preuve de la réussite du rituel. L'offrande de bière qui, dans un premier temps, a permis le départ du mort, sert une fois encore de médiation pour que se manifeste le signe de sa transformation. Tous les participants courent en criant vers son habitation. Là, l'aîné du village se tient face à sa case, un épi de mil en main. Il crache une gorgée de bière sur la panicule. Un instant après, une petite araignée blanche sort de l'épi ${ }^{7}$. L'apparition de cette arachnide témoigne pour les Koma de la présence du défunt et de sa satisfaction ${ }^{8}$. C'est la première manifestation du mort en tant qu'esprit. D'après Meek, cet animal doit être considéré comme un substitut du crâne. Son culte est couramment observé dans les sociétés qui conservent les

\footnotetext{
7 La lie de bière attire les araignées qui se logent dans les panicules.

- Dans les populations de la Bénoué, la réussite d'un rituel est toujours liée à l'apparition d'une araignée. Chez les Birom du plateau Bauchi, qui parlent une langue proche des Chamba, I'araignée joue une part importante dans beaucoup de rites (Meek 1931b, I:371). Pour J.G. Davies, l'araignée est l'équivalent de l'esprit d'un honme... Elle "contient le double de I'âme ou son complément" (1942: 106-107). On note des ressemblances entre les rites birom et koma destinés à guérir les jeunes enfants. One araignée posée sur la tête du malade signifie le retour de l'esprit égaré (cf. Davies: 123 et 353-361, et Dumas-Champion 1986: 56).
} 
crânes des ancêtres (Meek 1931b, I:367). Lorsque l'absence d'araignée vient dire la désapprobation des ancêtres sur la conduite de la famille, on veille à taire les dissenssions familiales et, une fois l'ordre rétabli, on renouvelle l'acte sacrificiel autant de fois qu'il sera nécessaire.

Lorsque s'achève la "bière de l'oubli" et que l'araignée se manifeste, on a l'impression d'assister à la fin d'un cycle, à l'aboutissement d'une longue mutation qui a fait du mort un esprit ancestral. Cependant, tant que ne sera pas organisée la "bière du crâne", l'esprit ne sera pas véritablement un ancêtre. La voie vers l'ancestralisation s'effectue, dans la conception koma, étape par étape, selon le modèle de vie d'un homme, marqué par une succession de stades initiatiques. Deux, voire trois années séparent généralement les deux cérémonies. La terre doit poursuivre son travail. Les Koma donnent, en effet, comme condition impérative à l'effectuation de la seconde cérémonie que le crâne soit libéré de toute trace de chair au moment de l'exhumation. Cette exigence n'est pas seulement justifiée par la crainte d'un reste qui rappelerait le statut de vivant du disparu; la présence de fragments de chair masquerait la véritable nature du crâne et nuirait au but recherché. Ces traces intempestives apporteraient la preuve d'un travail inachevé et viendraient contredire le caractère d'inaltérabilité du crâne. C'est la qualité de cette matière, quasiment indestructible, que l'on compare à la pierre et qui évoque l'intemporalité du monde des esprits, que ces populations mettent en avant lorsqu'elles font des crânes de leurs parents un objet de conservation privilégiée.

C'est au fils aîné, l'héritier` du crâne de son père et de sa mère, qu'incombe la décision d'organiser la cérémonie, au moment même où l'on invoque les ancêtres en vue de la prochaine saison agraire. Le mil destiné au brassage de la bière provient de la récolte du défunt dont une quantité de sept paniers a été soustraite à l'héritage des neveux utérins. Les parents, amis et alliés du défunt sont conviés à la fête, mais seul un petit comité se rend au cime-

\footnotetext{
9 Les Kona, matrilinéaires, transmettent leurs cultes en ligne patemelle.
} 
tière. Le fils n'assiste pas lui-même au prélèvement du crâne. Les aînés du village délèguent sur place le devin, géza, quelques anciens et quelques jeunes initiés qui partent avec trois pots de bière; les femmes qui le désirent - filles ou soeurs du défunt - peuvent se joindre au groupe.

La présence des femmes sur les lieux mêmes de la tombe peut surprendre quand on sait qu'elles en sont tenues à l'écart au moment de l'enterrement. Elles ne peuvent alors accompagner le corps qu'à mi-parcours sur le chemin du cimetière. La séparation se fait brutalement, au coup de sifflet du madi ${ }^{10}$. Les femmes doivent s'enfuir avant que vrombissent les rhombes, non sans abandonner les quelques objets du mort qu'elles portaient, et après avoir brisé les calebasses remplies des denrées provenant de son grenier. Il ne semble pas que ce soit seulement pour protéger les femmes du danger que représente la vue du mort au moment de l'inhumation - bien qu'il soit habituel de dissuader les jeunes, y compris les circoncis, d'assister à l'enterrement - mais aussi et surtout pour les tenir à l'écart de ce qui, dans le rite d'inhumation d'un homme, vient rappeler l'initiation du défunt aux cultes du vome (ou kéné), interdits aux femmes. En revanche, aucun rite secret ne semble avoir lieu pour l'exhumation du crâne. La présence des femmes est tolérée, peut-être même souhaitée? Ne doivent-elles pas être témoin, elles aussi, du changement d'état de leur parent ? Elles ont d'ailleurs parfois un lien à rompre avec celui-ci. Ainsi, une fille brisera sur la tombe la figurine de terre glaise, représentation de son père (ou de sa mère), qu'elle avait modelée de son vivant. L'effigie avait pour but de conserver ses parents en bonne santé. Au lieu de détruire cette statuette au moment de la mort, son propriétaire attend le prélèvement du crâne comme s'il cherchait, à travers la mise en rapport de ces objets de nature antinomique (l'effigie, représentation d'un vivant, étant brisée

\footnotetext{
10 Le madi, assistant du forgeron - celui-ci est aussi circonciseur et fossoyeur - a une fonction de dérision rituelle. En tant qu'éducateur des circoncis, il apparaît conme un garant de l'ordre masculin et exerce sa bouffonnerie tout particulièrement à l'encontre des fermes (Dumas-Champion 1986: 54).
} 
alors qu'on exhume le crâne, représentation de l'ancêtre) à souligner la mutation du défunt et à consommer la rupture du lien filial encore en jeu. Il semble bien que le temps de l'exhumation soit le moment choisi pour mettre un terme à toutes les relations qui unissaient les vivants au mort. C'est ainsi que les Chamba, dont les cérémonies funéraires présentent de nombreuses analogies avec celles des Koma, vont lever la malédiction qu'aurait proférée le défunt de son vivant, avant même de déterrer son crâne (Fardon 1982, II:73).

Revenons à l'exhumation. Généralement, l'opération ne se limite pas au prélèvement de la boîte cranienne mais concerne l'ensemble des os du squelette, afin de libérer la tombe pour un nouvel usage. Les Geme disent qu'"on fait sortir la personne", nek basunani. Les ossements une fois déterrés sont gardés sous un abri rocheux ou tout simplement poussés sur le côté de la fosse. Etant donné que les tombes sont réutilisées, il n'y en a pas plus de trois par cimetière. C'est seulement en cas de morts rapprochées qu'une nouvelle sépulture est creusée. Les initiés sont enterrés dans un lieu distinct de celui des femmes et des jeunes incirconcis qui sont, quant à eux, inhumés à proximité du village.

Avant d'être l'objet d'un examen attentif, le crâne est minutieusement nettoyé afin qu'aucun fragment de peau ou de tissu n'adhère plus aux parois osseuses. L'un des pots de bière est réservé à ce lavage qu'on doit peut-être considérer comme une première libation ${ }^{11}$. L'os crânien et les mâchoires dont l'apparence doit révéler la véritable nature du défunt sont alors analysés par le devin. La

"Chez les vere, le crâne est retiré, nettopé, lavé à la bière et barbouillé d'huile de karité (Meek 1931b, I:422). A propos de ce rite d'exhumation, A. Edwards écrit: "le lamz (fossopeur) et les autres participants se rendent sur la tombe avec trois pots de bière tout en soufflant dans une corne. Ils retirent la pierre plate qui ferme la tombe et prélèvent la tête. Ils lavent le crâne à la bière, boivent ensemble l'un des pots, versent le dernier sur la tombe puis enduisent la tête d'ocre" (Documents non publiés). Chez les Duru, "le forgeron prélève le crâne et le nettoie. Il le place ensuite dans une calebasse et le ramène chez lui. Là, il le nettoie encore une fois à fond et le peint de traits ocres, dont l'un correspond à la couture médiane et l'autre croise la prenière et longe l'arrière de l'os frontal" (Frobenius: 244). Cette cérémonie se déroule donc selon les mêmes modalités chez les Chamba, Kona, Vere et Duru. 
présence de trous minuscules apporte la preuve de sa sorcellerie ${ }^{12}$. Cet examen est décisif. Si, jusque là, le défunt avait été considéré comme un sorcier et si son crâne présente un aspect lisse et sans irrégularité, il est de mise de revenir sur ce jugement pour affirmer qu'il était, en fait, innocent. Il est vrai que les parents n'attendent pas trois années, le temps de l'exhumation, pour rechercher les signes susceptibles de les informer sur les causes de la mort. Ils commencent leur investigation le lendemain de l'enterrement. Les frères du défunt, ou le mari et le frère aîné de la défunte, se rendent au cimetière pour examiner la tombe. S'ils distinguent à proximité de la pierre tombale un trou de la grosseur d'un doigt, ils en déduisent que le mort était un "sorcier-anthropophage". S'il n'y a rien, ils pensent qu'il a succombé à une mort naturelle. Les Koma attribuent toujours la mort d'un sorcier à l'action des traitements anti-sorcellerie dont ils font un grand usage ${ }^{13}$. En revanche, il est difficile de savoir si un mort a été victime de sorcellerie.

L'état du crâne révèle toujours, semble-t-il, la véritable nature d'un individu, information que ne donne pas la singularité des traits du visage, qui permet seulement de différencier les individus les uns des autres. Rien dans l'apparence physique ne distingue un sorcier d'un autre homme - tout du moins en dehors des moments où il n'agit pas en tant que tel. En revanche, après la mort et l'effacement des formes du visage apparaît la véritable identité d'un individu. Le crâne, considéré comme le siège de l'âme, divulgue, de par les modifications de son ossature, la nature de l'esprit qu'il enferme. Doit-on comprendre que seul l'esprit malfaisant d'un sorcier peut

12 Chez les Fali, "les excroissances anormales au niveau des apophyses stylö̈des ou de la symphyse mentonnière" sont la preuve que le crâne abrite des "mauvais esprits" (Gauthier 1969: 175). Chez les Dowayo, la présence d'ecchondroses derrière la mâchoire indique que la personne concernée était un sorcier. Si ces excroissances pointues sont rouges ou noires, c'est signe que le sorcier a fait des victimes. Si elles sont brisées, le sorcier a été tué par une médecine contre la sorcellerie (Barley 1983: 111). Cette pratique, qui est largement attestée dans les sociétés de la Haute Bénoué, l'est encore dans d'autres régions, notament chez les Challa, voisins des Birom du plateau Bauchi (comunication personnelle de J.C. Muller).

13 Les Dowayo raisonnent de la même manière. "Quand les Dowayo, écrit Barley, déclarent qu'un tel a été tué par sorcellerie, ils veulent normalement dire qu'il était lui-mêrne un sorcier" (p 37). 
modifier cette matière supposée inaltérable qu'est le crâne? L'enquête demanderait à être approfondie. Nous ignorons d'ailleurs ce qu'il advient des crânes de sorciers. Deviennent-ils comme les autres un objet de culte? Chez les Fali, il semble que tous les crânes soient systématiquement frottés à l'aide d'une mixture ${ }^{14}$ destinée à éloigner les mauvais esprits (Gauthier: 175). Les Dowayo, quant à eux, nettoient le crâne avec des feuilles d'un arbre dit tarko ${ }^{15}$ dont la symbolique rappelle au défunt sa position de candidat à l'ancestralisation (Barley: 111).

Une fois examiné, le crâne est oint d'huile et d'ocre selon un traitement similaire à celui des vivants ${ }^{16}$. La prière prononcée par l'officiant ainsi que celles que son fils adressera à son père donnent quelques éléments d'exégèse sur ce rite auquel les Koma attachent la plus haute importance. "Au nom de ton fils", dit l'aîné des célébrants en s'adressant au défunt, "je soustrais ton crâne aux mauvais esprits en présence des hommes et du N'Da"17. Dans ses prières ultérieures, son successeur insistera encore pour rappeler l'acte essentiel dont il est l'instigateur. Il ira jusqu'à dire qu'"il s'est montré un homme en délivrant son père des esprits néfastes qui peuplent la brousse". On peut s'étonner d'une telle insistance pour un acte qu'il est tenu d'accomplir. Il est vrai que sans l'exhumation du crâne, le défunt ne pourra jamais être ancestralisé. Aussi, pour un homme ou une femme décédé sans laisser de descendance, un frère se chargera de mettre leur crâne "à l'abri", selon l'expression usitée par les Bamileke et qui traduit le même objectif (Pradelles de Latour 1986: $156^{18}$ ). Extraire le crâne de la terre et en faire un objet de culte, c'est donner au défunt rang d'ancêtre. L'héritier qui

\footnotetext{
14 Ce mélange à base de cissus quadranqularis est également utilisé chez les Roma pour expulser le mort (cf. plus haut).

1s Arbre non identifié par l'auteur.

${ }^{16}$ Les Koma ont l'habitude de s'enduire la tête d'huile ocrée. Un informateur vere de $A$. Edwards explique cette onction comme un moyen de conserver le crâne "afin qu'il ne se brise pas".

17 Prière formulée par les Gumbe. N'Da désigne la divinité suprême.

1 B "Pour qu'un défunt devienne ancêtre et passe ainsi du côté des dieux, il faut que son successeur détache son crâne du squelette un an ou deux après l'enterrement. Les Bangwa disent que les "têtes" doivent être mises à l'abri de la pluie".
} 
achève ainsi le cycle funéraire est quitte auprès de son géniteur : en le faisant accéder au statut d'ancêtre, il intervient, pourrait-on dire, dans sa "re-naissance". S'il insiste sur son geste et sur la réciprocité en jeu dans cet engagement, c'est dans l'espoir d'être, en quelque sorte, payé de retour. Telles sont les considérations que les Chamba Leko avancent aussi dans leurs prières. "Aujourd'hui", dit un vieil officiant en s'adressant au défunt, "nous sommes venus te conduire jusqu'à la maison de ton fils. Car, ce n'est pas sa volonté que tu restes abandonné en brousse. Peux-tu donc prendre soin de lui comme il prend soin de toi. Veille à ce qu'un malheur ne l'accable pas" (Meek 1931b, I:382). L'exhumation du crâne apparait donc comme l'intervention première, l'acte princeps, qui fonde la relation aux ancêtres.

Le crâne, déposé dans une poterie, a été rapporté au fils aîné, demeuré chez lui. A cause du danger encouru, le transport est toujours effectué par les vieillards que ce soit chez les Koma, les Vere, les Chamba ou les Dowayo. Ces derniers croient que le porteur "en mourra quelques années plus tard" (Barley: 71). C'est pour les mêmes raisons que, chez les Chamba, un fils ne manipule jamais le crâne de son père; un vieil homme s'adressera au défunt à la place du jeune héritier. Cette précaution n'est pas de rigueur pour les Koma. Le fils accomplit lui-même le premier acte sacrificiel qui installe son père parmi les ancêtres et qui, du même coup, instaure entre eux une nouvelle relation. La prière qu'il prononce, en égorgeant une chèvre dans la cour intérieure de son habitation, "Père! je te donne ton sac", fait référence à la sacoche dont un Koma ne se sépare jamais dans ses déplacements. On dit que "les ancêtres doivent voir arriver le nouveau venu avec son sac" (Dogari 1984: 69). Cette formule rituelle marque l'ultime voyage du défunt et son accession à l'ancestralité. Dès lors, lorsque le fils sort, muni d'une louche du sang de la victime, pour procéder à la première libation sur le crâne déposé au pied d'un arbre, il s'adresse à son père en tant qu'ancêtre: "Père! je t'ai donné ton sac. Je te prie de faire en sorte que je sois chanceux dans la vie, que j'obtienne une bonne récolte et que j'aie des enfants". L'exhumation du crâne, qui marque 
l'aboutissement du procès d'ancestralisation pour le défunt, achève, par voie de conséquence, le principe filiatif. Désormais, l'héritier du crâne, en tant que nouveau chef de famille, prend en charge les sacrifices familiaux dédiés aux ancêtres.

L'assemblée réunie pour la cérémonie commence à boire "la bière du crâne" non sans offrir une première libation au nouvel ancêtre, selon le cérémonial déjà établi lors de "la bière du mort". La viande de chèvre qui a été cuisinée par les femmes est partagée entre les participants. Les danses et les chants qui accompagnent la fête se poursuivent jusqu'au soir, au moment où un petit groupe d'hommes s'en retourne, non sans cesser de chanter et de danser, déposer le crâne dans l'arbre qui sert de cimetière et qui reçoit tous les crânes des hommes disparus ayant appartenu à la même communauté. Les poteries qui leur servent de réceptacle sont placées en équilibre sur les branches. Les crânes des femmes sont entreposés dans un autre lieu. Plus tard l'héritier pourra construire un grenier non loin de son habitation pour y déposer le crâne de son père. L'inauguration donne lieu à une libation de bière sur le crâne et au sacrifice d'un animal, chèvre ou poule, dont le sang est versé sur la boîte crânienne. Si la victime est une volaille, on colle le duvet sur le sang coagulé. Une fois enfermés dans le grenier ou à l'abri dans une poterie, les crânes ne sont plus l'objet d'aucune manipulation rituelle jusqu'à l'année suivante ${ }^{38}$ où un cycle de cérémonies est organisé entre tous les détenteurs de crânes. Les reliques sont alors portées sur les lieux de l'habitation où chaque propriétaire les enduit d'une bouillie de mil en nommant tous ses ancêtres du côté paternel et maternel afin de les convier au sacrifice, doge kela, qu'il dédie à son père (et/ou à sa mère). La prière qu'il formule est une demande de prospérité pour la nouvelle saison agraire. Elle est semblable à

19 A la différence des Koma qui n'entretiennent pas un contact intime avec les crânes de leurs parents - tout au moins de nos jours - les Vere placent (ou plaçaient autrefois) le crâne de leur père près de leur tête durant la nuit et "font des prières fréquentes au crâne" (Meek 1931b, I:422). Dans des documents récents fournis par A. Edwards, il est dit que tous les crânes du lignage sont gardés ensenble dans le luuk, lieu sacré, exactement come chez les Chamba et les Dowayo. 
celle des autres sacrifices qui inaugurent les différentes phases de la récolte: les prémices, la moisson et le battage.

Bière ou bouillie de mil, sang d'une victime ou onction de traits ocres, sont les seuls éléments auxquels les Koma recourent aussi bien pour régénérer leurs objets et instruments cultuels ${ }^{20}$ que pour vénérer les crânes de leurs ancêtres et traiter rituellement le front des vivants, qu'il s'agisse d'initiés, d'officiants ou de malades. Ce type de marquage met en évidence une corrélation entre le traitement du crâne et la circoncision ${ }^{21}$. Ce sont, pour les Koma, les symboles même de la vie: la bière de mil ou la bouillie de mil germé est associée à la semence masculine, l'ocre ou le sang représente le sang féminin. On retrouve, dans tous les rites koma, ce même schéma opératoire mettant en jeu les termes de la procréation ${ }^{22}$.

\section{La conservation d'objets en rapport avec l'ancêtre}

Le crâne, libéré de toute trace de chair qui pourrait rappeler l'identité passée du défunt apporte, de par sa matière dure et inaltérable, la preuve de l'ancestralisation du parent. Si reste de squelette

20 Les objets cultuels dits kene ou vome, ainsi que les pierres dressées, les trophées des animaux tués à la chasse ou les massacres des taureaux abattus en l'honneur de la belle-mère, sont pareillement traités.

${ }^{21}$ Ainsi, le novice qui accède à l'ultime stade de son initiation est marqué à l'ocre d'un long trait qui part du sommet du crâne jusqu'à l'arête nasale; de part et d'autre de cette ligne, deux traits blancs à la farine de mil complètent ce tracé. Les circonciseurs reçoivent la même onction, avant la cérémonie. Un malade est soigné de manière identique. Ces marques ne sont pas spécifiques aux Koma. A propos de Mapeo, Fardon rapporte qu'avant. de circoncire les néophytes, "on touche le front du premier garçon de chaque clan participant à 1 'aide de couteaux de circoncision pour ensuite leur couper le prépuce avec n'importe quel couteau pointu" (1980, II:38). Cette mise en vedette du front a lieu aussi lors de la cérémonie d'entrée au culte yaagumeni (les propriétaires de ce culte sont les Yambu, patriclan d'origine Vere), "les aînés font mine d'inciser chaque adolescent en traçant, à l'aide d'une flèche, une ligne du sommet du crâne jusqu'au milieu du front" (Fardon, II:42). Meek relate encore, au sujet des Chamba, un rite sacrificiel où sont traités conjointement le crâne de l'ancêtre qui tient le rôle d'agent pathogène et le front du malade (1931b, I:382).

22 Nous ne disposons pas d'informations suffisantes pour interpréter la signification du tracé dont les crânes font l'objet aussi bien chez les Kona que dans les autres populations de la région (Duru, Vere, Dowayo), à savoir une longue ligne médiane de bouillie de mil gerné barrée par deux lignes transversales de couleur ccre. Rappelons que tous les objets cultuels (instruments de musique, couteaux de circoncision) sont affectés de ce signe. 
il y a bien, la transformation qui s'est opérée pendant le travail du deuil dans l'imaginaire des deuilleurs, en corrélation avec un travail de décomposition de la matière, a recréé une "image ancestralisée "du parent, image concrétisée par l'exhumation du crâne, alors visible aux yeux de tous, et qui n'a plus rien à voir avec le souvenir du disparu. Le crâne n'évoque plus l'individu, il est la représentation de l'ancêtre. Mais, dans ce cas, que penser des autres objets qui sont mis en rapport avec le défunt en vue d'une conservation? Sont-ils eux aussi une représentation de l'ancêtre?

Le mourant n'a pas encore trépassé qu'on recueille son souffle en frôlant sa bouche à l'aide de deux plumes qui sont ensuite piquées dans une petite corne obturée à la cire ${ }^{23}$. Cette corne, dite vo'te, symbolise l'âme du mort. Chaque défunt, homme ou femme, est représenté par un tel objet. Le vo'te peut être gardé par l'héritier du crâne, chez lui, à l'intérieur d'un sac qui pend d'une solive de l'habitation, ou avec les autres vo'te du clan, rassemblés dans une cavité rocheuse, à l'extérieur du village. Cette corne reçoit un traitement rituel identique à celui du crâne. De même qu'on organise une fête annuelle pour les crânes, on célèbre les vo'te chaque année. Par la médiation des vo'te, les ancêtres exercent leur protection sur les membres de leur famille. L'oubli ou la négligence de ceux-ci les fait se manifester comme agents pathogènes. La virulence de la maladie est d'autant plus forte que l'ancêtre est dans une relation de proximité avec le malade. Chaque corne se distingue par sa taille et sa forme, de telle sorte qu'aucune erreur n'est possible sur l'identité du vo'te dont le devin révèle la part de responsabilité dans la maladie. Pour rétablir l'ordre, on lui sacrifie une chèvre et on arrose le vo'te d'une libation de bière. Ainsi, cette corne est une autre représentation de l'ancêtre. Il nous est difficile d'appréhender, dans l'état actuel de nos connaissances, la différence existant entre ces deux objets médiateurs.

23 Cette pratique est attestée dans deux groupes koma: les Geme et les Gumbe. Nous ignorons si elle existe chez les Goonu. 
On ne saurait dissocier la pratique de conservation des crânes de celle qui consiste à conserver d'autres parties du corps. Certains groupes chamba qui, en dehors du crâne, conservent des dents, des rognures d'ongles, des os du bras et d'autres objets les identifient sur le plan rituel en les désignant d'un même terme: uri, "crâne". Qu'il s'agisse de l'autel du patriclan - le seul à être composé de crânes - ou de l'autel du matriclan qui utilise, en remplacement, des cols de poterie, les cérémonies effectuées auprès de ces autels sont désignées d'un même terme, ten uri, "la prise du crâne", et leur déroulement est identique. Alors qu'à chaque mort, l'autel du patriclan s'enrichit d'un crâne, un pot nouveau est ajouté à l'autel du matriclan (ou, à défaut, le cou d'une jarre de bière cassée) (Fardon 1980, II:73). Ces petits pots qui représentent les défunts reçoivent des offrandes de bière exactement comme des crânes. On peut se demander dès lors si l'équivalence que posent les Chamba entre le crâne et d'autres parties du corps ne repose pas sur un trait qui serait commun à ces "objets", à savoir leur inaltérabilité. Qu'il s'agisse des ongles, des dents ou des os, ils échappent à la décomposition du cadavre et apparaissent, tout comme le crâne, associés à l'intemporalité des esprits ancestraux.

Pour expliquer l'identité rituelle qu'établissent les Chamba entre le traitement du crâne et celui d'autres objets cultuels, il est indispensable de faire référence à la fonction de l'institution du voma parmi les populations de la Haute Bénoué. Ce culte, d'origine chamba, est largement diffusé dans la région puisqu'il est attesté jusque chez les Jukun sous le nom de buhor (Dumas-Champion 1987). Chez les Koma, il y a une corrélation étroite entre la structure de ce culte et l'organisation sociale et politique de la société. Les détenteurs de pouvoir au sein de chaque communauté - chacune d'elles étant politiquement autonome - sont les propriétaires du $v$ vme $^{\mathbf{2 4}}$. Chaque individu mâle accède au cours de sa vie à plusieurs étapes qui l'initient progressivement à la connaissance du vome. Au

24 L'institution considérée, dénommée du terme générique vơma en langue chamba leko, jubi en daka, vome en gema, inclut un grand nombre de cultes associés à des maladies ou à d'autres malheurs. 
terme de cette initiation, à un âge avancé, l'homme est intégré au collège des anciens qui, aux côtés du chef rituel, dirige la communauté. Des rites funéraires, spécifiques au vome, complètent le deuil ordinaire. C'est dans ce cadre que le forgeron et les chefs rituels sont enterrés avec des rhombes sous les aisselles. Ces sacra sont restitués au culte lors de l'exhumation du crâne.

Quelle est donc la finalité d'une telle inhumation? Dans un clan chamba d'origine vere, les Yambu, on ensevelit un homme avec un morceau de trompe en calebasse, qui est l'instrument rituel par excellence, et on place une petite pierre dans la bouche d'une femme. Ces objets, retirés au moment de l'exhumation du crâne, sont désignés par le même terme : uri, "crâne" (Fardon, II:41). Alors qu'on eût pu croire, eu égard à l'importance de l'institution du voma, que le culte du crâne était secondaire par rapport à celle-ci - l'objet crâne étant traité comme les objets sacrés du vome - cette assimilation terminologique nous invite à renverser la proposition. Les propos recueillis au début du siècle par Frobenius confortent cette idée. Chez les Daka, "les instruments cultuels les plus précieux du jubi (équivalent du voma) sont avec les rhombes, langa, des bracelets en fer auxquels pendent des grelots et des plaques en fer, dozi. A la mort d'un kameni, prêtre du jubi, le forgeron fabrique aussitôt un grelot qu'on placera dans la tombe du défunt. Puis, lorsqu'on estime que le cadavre s'est décomposé, on ouvre la tombe pour y prélever le grelot. Il est nettoyé au moyen d'un médicament, puis on l'accroche sur le dozi à côté des autres grelots". Tout se passe comme si, par ce séjour sous terre, tel un crâne, le grelot acquérait, en assistant au long processus de décomposition, quelque chose du principe de l'ancestralité puisqu'on s'adresse aux dozi comme aux "grands-pères". D'autre part, c'est bien de représentation d'esprits ancestraux qu'il s'agit puisqu'on dit "du bruit de ces sonnailles que ce sont les voix des ancêtres" (Frobenius 1925/1984: 73).

Voyons ce qu'il en est des autres représentations et notamment des pierres qui sont les symboles substitutifs privilégiés des crânes tant chez les Jukun que dans les autres populations de la Bénoué. La manière dont ces pierres sont censées acquérir leur valeur, chez 
les Fali de Ngoutchoumi, doit retenir notre attention. Il est, une fois encore, question de mise en contact avec le cadavre mais sans inhumation. "Placée entre les cuisses du mort, la pierre prend de sa puissance sexuelle, que les vivants conserveront ainsi et pourront lui emprunter par des sacrifices... Celle-ci est ensuite frappée à l'aide d'un petit bâton afin d'inviter l'esprit du défunt à y pénétrer" (Gauthier: 169-170). Ces pierres vont être sollicitées à la place du crâne qui, bien que déterré, n'est pas conservé à des fíns de culte. Il est inhumé définitivement une seconde fois. Chez les Fali du Tinguelin, Jean-Paul Lebeuf signale que les pierres "qui représentent les hommes sont accompagnées de reliques, mâchoire inférieure (le crâne étant conservé à part), gourde pour le tabac à priser et pointes de flèches" $(1961: 63)^{25}$. La forme des pierres varie selon le sexe du défunt: "plates et angulaires pour les hommes, meules courantes hors d'usage pour les femmes". L'équivalence pierre/crâne/ancêtre apparaît ici sans équivoque.

Citons encore l'exemple des Dowayo qui exhument les crânes de leurs morts, à l'exception de ceux des chefs de pluie. La tête manquante est remplacée par une pierre pour le chef de pluie de Kpan, et par une poterie pour celui de Mango. A défaut de pierre, les pots de terre cuite sont les symboles les plus couramment substitués aux crânes. Cette pratique est observée notamment chez les Yungur du Nord et chez les Jibu, qui retiraient autrefois les crânes de leurs morts et les plaçaient dans un pot avant de les déposer sur une branche fourchue faisant office d'autel puis, à une époque plus récente, sans procéder à l'exhumation, ont simplement fait usage de poteries (Meek, II: 464 et 516). Chez les Chamba, les pierres font directement référence au monde ancestral. On dit des pierres aux formes originales qu'elles sont l'oeuvre des ancêtres. A ce titre, elles sont ajoutées aux autres objets du culte voma. On dit aussi du rite d'intronisation des chefs rituels - qui consiste dans le fait d'asseoir l'impé-

25 Dans un article ultérieur (1987), Lebeuf précise que la conservation des maxillaires inférieures des défunts n'est observée qu'au Tinguelin. 
trant sur la pierre du voma - que c'est un moyen pour le chef d'entrer en relation avec le monde des ancêtres (Fardon II: 31 ).

L'identité de traitement entre le crâne et les objets cultuels amène à penser que les traitements rituels liés au crâne offrent à ces populations un schème de pensée pour construire toutes les représentations de l'ancestralité. Le crâne, partie inaltérable du corps, apparaît comme le paradigme des objets cultuels du vome. Il en résulte que le culte du vome, institution largement répandue, est subordonné par rapport au culte des crânes qui recouvre la même extension géographique.

\section{De la circoncision à l'ancestralité}

Le cas des Dowayo que nous allons présenter maintenant nous aidera à proposer une interprétation concernant la pratique de la décollation des crânes. Les matériaux exposés par N. Barley (1983) mettent en évidence le fait que la circoncision a valeur de paradigme dans cette société. Ce trait, pensons-nous, est récurrent chez les Chamba, Duru, Pape, Voko et Koma; l'exemple des Dowayo nous sera d'autant plus précieux qu'il permet de préciser un enjeu commun à ces populations.

Que l'initiation soit remise en scène à l'occasion des cérémonies funéraires n'a en soi rien d'original mais la constante référence que les Dowayo y font donne une autre dimension à cette pratique. En tant que rite de passage, l'initiation apparaît servir de modèle pour penser les étapes successives qui marquent l'accession du défunt à l'ancestralité. Si l'on considère le traitement du cadavre, celui de l'arc et enfin celui du crâne, on constate que le mort est placé, à chacun des stades de son cheminement vers son statut d'ancêtre, dans la position d'un candidat à la circoncision. Ce modèle ne s'applique pas seulement au mort, il est encore le système auquel on fait référence pour séparer les deuilleurs du défunt puisque les veuves sont, au cours des phases marquantes du travail de deuil, mises dans une position de circoncis. De plus, nous verrons comment 
les compagnons de circoncision du mort interviennent aux côtés des agnats ou des bouffons pour favoriser son passage au statut d'ancêtre. Alors que dans la première partie de cet article, nous avons mis en évidence le fait que l'exhumation du crâne était l'acte fondateur du culte des ancêtres, nous allons maintenant examiner à travers le déroulement de la "cérémonie des crânes", ultime phase du cycle funéraire, l'articulation entre la circoncision et l'accession au statut d'ancêtre. L'habitat où s'achève le trajet du défunt, alors que son crâne y fait cérémoniellement son entrée, a valeur emblématique pour les Dowayo. Dit "la maison des crânes", ce lieu est non seulement celui des ancêtres mais aussi le site de référence que les vivants ont choisi pour signifier leur cohésion et l'origine de leur appartenance. En effet, la plus grande unité sociale est "le groupe de la maison des crânes" qui réunit tous les descendants en ligne agnatique, hommes et femmes ${ }^{28}$. Cette "maison" est bien plus qu'un simple "reliquaire". C'est le lieu où s'organisent et d'où partent l'ensemble des cérémonies scandant la vie d'un Dowayo ainsi que les rites afférents au traitement des maladies. C'est, dans cette société patrilinéaire, le centre de la vie sociale. Les décisions religieuses y sont prises collectivement par les membres les plus anciens. A la tête de ce groupement social se trouve généralement le "prêtre de la maison des crânes". Maître des cérémonies, il est aussi le gardien du lieu et doit veiller non seulement sur les crânes des ancêtres mais aussi sur les objets cultuels sacrés comme les rhombes ou les pierres destinées à assurer la fertilité du mil (Barley: 54). Il est aidé dans sa tâche par des bouffons rituels qui ressemblent trait pour trait aux madi des Koma.La "maison des crânes" et ses environs proches circonscrivent un territoire hautement sacralisé et, à ce titre, interdit aux femmes. On raconte que "leur odeur mettrait en colère les esprits et qu'elles deviendraient stériles". Bien que les crânes des ancêtres féminins soient assaciés à ceux des ancêtres masculins lors des cérémonies, ils sont entreposés dans un lieu

26 La résidence est patrilocale mais les femmes mariées sont enterrées dans leur village natal et leur crâne intégré à la "maison des crânes" de leur père. C'est aussi le cas chez les Vere. 
distinct de celui des hommes, généralement sous un arbre. La séparation des sexes est chez les Dowayo, comme dans l'ensemble des peuples de la Haute Bénoué, un trait fortement marqué qui s'exprime par l'exclusion des femmes d'une grande partie de la vie rituelle. Seuls les hommes initiés ont donc le droit de pénétrer dans cette "maison" -symbole de l'ordre masculin- dont le premier accès constitue pour le circoncis l'étape décisive qui fait de lui un homme à part entière. Après sa mort, l'intégration de son crâne à la maison des ancêtres est la phase ultime qui marque son accession à l'anscestralité. Cette dernière "entrée" dans la "maison des crânes" est pour les Dowayo l'occasion d'une cérémonie qui rassemble les plus grandes foules, où sont conviés parents, alliés, amis et étrangers. Célébrée à n'importe quel moment après la mort, elle est souvent le résultat d'une pression émanant d'un défunt qui se manifeste d'abord sous forme onirique, puis par une maladie. L'agnat du mort ainsi persécuté se voit contraint d'organiser la cérémonie en collaboration avec ses frères, en demandant la contribution financière de ses autres parents et de ses alliés. En raison des dépenses somptuaires que cette fête nécessite en mil et en viande - des bovins et des caprins sont abattus en nombre - les crânes des morts récents appartenant à la même maison sont traités ensemble, qu'il s'agisse d'homme, de femme ou de forgeron (Barley: 99). Ces frais sont compensés par le prestige non négligeable dont les organisateurs bénéficient aux yeux du public.

Autrefois, lorsqu'une cérémonie des crânes allait être organisée, les agnats $\mathrm{du}$ mort et ses frères de circoncision tendaient une embuscade pour tuer un étranger. Cet homicide se déroulait sur un mode guerrier. Les tueurs, de retour au village, érigeaient un nouveau dõre - sorte de pieu fourchu qu'on dresse en cas de meurtre ou lors de l'abattage d'un fauve - et entonnaient un chant de guerre (p.62). Arrêtons-nous un instant sur le traitement de la victime. Son sexe importait peu, mais elle devait être habillée, après sa mort, d'un chapeau rouge de Peul. Ce trait vestimentaire fait directement référence au mythe d'origine de la circoncision et à la vieille femme peule qui fut battue à mort par les Dowayo après qu'ils lui eurent 
emprunté cette pratique ${ }^{27}$. Barley ne dit pas pourquoi l'adoption de cette opération, qui a valeur civilisatrice, impliquait nécessairement un meurtre. Mais on peut penser que ce récit est la métaphore de l'opération même de la circoncision. L'ablation du prépuce - auquel on confère un caractère féminin- est représentée par l'exécution à coups de bâtons d'une femme peule. La légende de circoncision des Duru présente une variante. Cette fois, les forgerons s'approprient cette pratique non sans avoir tué la femme qui fit subir à son mari une opération dont elle fut le témoin chez les $\operatorname{singes}^{28}$. La connaissance ne va pas sans une forme de mise à mort. C'est bien en vertu de ce principe que les circoncis, pour être initiés au secret des hommes, sont bastonnés lorsqu'ils pénétrent pour la première fois dans la "maison des crânes". Cette séquence initiatique, dans sa mise en scène, est commune à la majorité des populations de la Haute Bénoué.

La circoncision est encore le paradigme d'autres transformations qui concernent non pas l'homme mais le végétal. Le battage du mil d'un "vrai cultivateur" se célèbre comme s'il s'agissait d'une vêritable circoncision. Il a lieu de nuit, sans la participation des femmes. Les bâtons que les hommes utilisent sont en bois de gokoh, semblables à ceux que portent les circoncis. Durant le battage, les hommes entonnent le chant de circoncision (p.65). Le mil, de nature féminine, frappé à mort à l'aide de bâtons, est "circoncis". La perte de son enveloppe donne les grains mâles.

27 voici le récit de ce mythe: "Une vieille ferme peule avait un fils qui était malade. Il s'était blessé en courant dans l'herbe, silkoh. Son penis enfla et devint purulent. Sa mère prit un couteau et lui coupa le prépuce, ainsi l'enfant guérit. Son pénis embellit. Elle circoncit de la même manière son second fils. Un jour qu'elle se promenait dans un village dowayo, ceux-ci virent que c'était bien. Ils adoptèrent la circoncision et la battirent à mort. Voilà l'origine de cette pratique car les Dowayo ne connaissaient pas la circoncision. Ils interdirent aux femmes $d^{\prime} y$ assister, à l'exception des femmes peules" (Barley:60).

2* "Une fenme se promenant en brousse vit un singe ccupant l'extrémité du prépuce de son petit. La fenme raconte le fait à son mari et le persuade de subir la même mutilation dans leur intérêt commun. Elle circoncit son mari au moyen d'un couteau emprunté à un forgeron. Ces derniers, avertis ultérieurement de l'événement par le premier circoncis, tuent la femne et reprennent le couteau. Ce seront eux désormais qui pratiqueront la circoncision, qu'ils auront apprise de la ferme qui elle-même la tenait du singe" (Podlewski 1971:29). La révélation de la circoncision par les singes est un trait récurrent dans d'autres populations plus lointaines comme les Mundang (Adler 1987:149) et les Masa (Champion 1977:249). 
Mais s'il n'y a là qu'un processus métaphorique, pourquoi un véritable meurtre devait-il être autrefois commis quand une simple mise en scène suffit aujourd'hui? Cela tient sans doute au contexte de guerre dans lequel vivaient les Dowayo, toujours en conflit avec leurs plus proches voisins, tout particulièrement les Peuls. Le traitement réservé au crâne de la victime témoigne de ce long passé conflictuel. Alors que le crâne du parent est conservé, la victime était décapitée et sa tête broyée. Quand on sait l'importance attachée à la conservation du crâne, ce meurtre équivalait à une double mise à mort, réduisant à néant la possibilité pour la victime d'accéder un jour au statut d'ancêtre. L'enterrement devait avoir lieu en brousse, sous une pile de pierres. Il présentait les mêmes caractéristiques que l'enfouissement des feuilles de borassus portées par le bouffon lorsqu'il interprète le personnage de la vieille femme peule - sa traîne de feuilles est alors frappée à coups de bâtons par les hommes. L'enfouissement $d u$ pansement d'herbe qui protège la blessure des circoncis est également à rapprocher de cet enterrement. L'équivalence de traitement entre les restes de la victime - fictive ou réelle - et ceux de la plaie, souligne le fait que la circoncision a partie liée avec un meurtre. De même qu'après l'enfouissement de leur pansement, les initiés prennent un nouveau nom qu'ils ne prononceront qu'au moment de leur entrée dans la maison des crânes, en réponse aux anciens qui les interrogent sur leur identité; de même, après l'inhumation de la victime, on appelle le défunt par son nom pour célébrer son entrée dans la maison des crânes, aux côtés des ancêtres.

Ce meurtre rituel qui s'apparente à une chasse aux têtes n'a cependant pas pour finalité l'acquisition d'un trophée mais la destruction d'un crâne. Outre la gratuité du crime qui, disent les Dowayo, augmente la fertilité générale, pourquoi le crâne d'un ennemi doit-il être détruit pour que le crâne d'un parent trouve place aux côtés de ses ancêtres? Cette épreuve que les agnats et les "frères d'initiation" du mort accomplissent en son nom doit être rapprochée de la mise en scène du rite de circoncision. La perte du prépuce est censée 
résulter d'un combat victorieux contre un léopard qui se jette en rugissant sur le candidat. Le circoncis qui sort vainqueur de cette lutte rythmée par un chant de guerre aura la taille ceinte d'une peau de léopard et l'on dira des initiés qui ne reviennent pas qu'ils ont été dévorés par ces fauves. Les frères du défunt qui doivent tuer sont dans la position du léopard qui circoncit; et l'étranger est une victime qui échoue devant l'épreuve de la circoncision.

Pour le circoncis, l'entrée dans la "maison des crânes" marque le passage à l'état d'homme; pour le défunt, l'installation du crâne dans cette "maison" fait de lui un ancêtre. Entre le moment de sa mort et cette intégration finale, il est censé passer par une succession d'étapes qui le replacent dans une position de circoncis. D'emblée, son cadavre est traité comme tel. Le lieu où il est préparé en vue de son enterrement, les soins qu'il reçoit, l'accoutrement dont on l'affuble sont autant de traits qui l'identifient à un candidat à la circoncision. Il est transporté dans l'abri des défunts pour y être toiletté à l'endroit même où les néophytes sont rasés et vêtus. Ses pieds sont liés avec une herbe (sole) de la même variété que celle que l'impétrant attache autour des chevilles et qui est coupée juste après l'ablation du prépuce. Si lui ou son père était un meurtrier, on lui met dans la bouche ou sur les épaules de l'écorce rouge diiblyo du même type que celle dont le circoncis a la taille ceinte. On l'habille d'une robe bleue et blanche, et d'un chapeau rouge, exactement comme la vieille femme peule. Puis il est successivement enveloppé dans la peau d'un grand nombre de bêtes qui sont immolées pour l'occasion. Autrefois, on n'hésitait pas à abattre la moitié du troupeau du mort. Le cadavre, ainsi enveloppé de plusieurs épaisseurs de peaux de boeufs et de vêtements funéraires, a l'aspect d'un énorme ballot en forme de poire. (A la veille de la circoncision, l'adolescent, tel un mort en puissance, porte lui aussi un vêtement funéraire ainsi que des peaux de chèvre et de mouton). La peau qui sera au contact du corps doit être celle d'un taureau au corps parfait. 
Si le défunt est un chef il faut choisir le meneur du troupeau ${ }^{29}$. On enduit d'huile et d'ocre rouge la tête et la robe de l'animal, comme le cadavre l'a été précédemment. Couché sur le flanc à côté du mort, on lui tranche la gorge. La peau est ensuite placée sur le cadavre, la tête sur celle du mort. Le corps est enfermé dans cette peau qui est cousue avec un fil végétal bosohse. (Une ou plusieurs années plus tard, on utilisera les mêmes liens pour habiller le crâne à l'occasion de son entrée dans la maison des ancêtres). La tête du défunt est traitée à part. Elle ne sera pas recouverte de cette même peau, mais de celle d'un bouc. Le choix de cet animal rappelle le statut de circoncis du mort. En effet, le sacrifice qui doit augurer $\mathrm{du}$ bon déroulement de l'initiation concerne l'immolation d'un bouc dont le sang est versé sur une variété d'herbe ( silkoh) qui, souvenons-nous, blessa le pénis du fils de la vieille femme peule. Dans le contexte de la circoncision, cette victime sacrificielle est la doublure de l'impétrant. Lors des funérailles, la peau de cet animal, qu'on appelle "le bouc nu", sert à recouvrir la tête du cadavre. Fourni par les agnats du mort, il est égorgé par son frère d'initiation qui enduit le cadavre de ses excréments. Ce badigeonnage est-il une brimade propre aux circoncis? Barley ne rapporte rien de semblable. En revanche, une telle épreuve existe chez les Koma où les bouffons, chargés de l'éducation des néophytes, n'hésitent pas à mater les "fortes têtes" en les bombardant d'excréments. Mais revenons à ce traitement bien particulier. Le maître de la maison des crânes et ses assistants jettent, en plus, sur le cadavre une substance végétale appelée "l'excrément du prêtre" ${ }^{30}$ qui est fabriquée à partir d'une écorce yaahutu pourrie dans l'eau. Lors du rite d'installation de l'arc, on attachera un morceau de cette écorce à l'arme du mort. Enfin, le jour qui clôture la cérémonie des crânes, on décorera le

29 Les Dowayo, qui élèvent une race de petit taurin, entretiennent des relations symboliques étroites avec leurs bovins. Barley donne quelques exemples concernant l'identification entre un home et son troupeau (19 et sq.).

30 Barley désigne du terme sorcerer toute une classe d'officiants rituels conme le maître de la maison des crânes, les circonciseurs et mêmes les bouffons sacrés. 
toit de son habitation avec ce même yaahutu. Cette matière est particulièrement réservée aux hommes qui tiennent une place importante dans la société. Les meurtriers ou les chasseurs de léopard en portent en permanence autour de la taille et leur fils doit en mordre durant la circoncision. A leur mort, ils sont enterrés avec un fragment d'écorce dans la bouche. Aux funérailles d'un circonciseur, ses pupilles portent des jupes en écorce et son corps est recouvert de cette même matière (p.116 note 30$)$. Enfin, pour la récolte d'un "vrai cultivateur", on place du yaahutu dans le premier tas de mil moissonné ( $p .117$ note 39 ). Cette substance connote l'idée de noirceur, de saleté, de nudité, dit Barley; mais comment ne pas associer aussi cette matière putride à la mort et à la décomposition du cadavre? Il reste, cependant, que ce traitement qui met en jeu des éléments de nature excrémentielle est d'autant plus achevé qu'il s'adresse à des hommes survalorisés d'un point de vue rituel. L'explication réside, une fois encore, dans la symbolique de la circoncision qui permet de comprendre la valeur conférée aux excréments qu'ils soient de nature animale ou végétale.

Dans la version réservée aux femmes, l'opération alléguée ne concerne pas l'ablation du prépuce mais l'occlusion de l'orifice rectal à l'aide d'un morceau de peau de vache (p.25). Ce discours opère donc un déplacement du pénis à l'anus. Cette fiction rappelle celle qui avait cours chez les Chagga du Kilimandjaro au XIXeme siècle. Les jeunes garçons subissaient une initiation au cours de laquelle on était censé leur suturer l'anus. Selon ce récit, les hommes adultes en âge de procréer ne déféquaient jamais (Moore 1976: 357). C'est aussi ce que les Dowayo veulent faire entendre aux femmes. Mais, quand les hommes ont la diarrhée, celles-ci ne manquent pas de leur faire remarquer que: "leur peau doit être pourrie" (Barley: 26). La suturation, chez les Chagga, connote la fécondité masculine dont la nature "fermée" évoque celle de la femme enceinte, alors au summum de sa fonction procréatrice. Le sang menstruel qui indique la nature "ouverte" de la femme est alors "absorbé". Les hommes qui sont censés "absorber" leurs excréments seraient donc perpétuellement "enceints". Il ne semble pas que les Dowayo aient développé 
une telle spéculation. Cependant, les séances de défécation collectives, les pets tonitruants, constituent toujours, dit Barley, "une allusion à la réalité de la circoncision". C'est ce qu'expriment les circoncis qui, à leur retour au village, s'adressant aux femmes, évoquent en chanson la mutation dont ils ont été l'objet: "Jusqu'à présent, nous avons dormi ensemble; maintenant, je vais péter dans ma case et vous allez péter dans la vôtre". La circoncision des populations voisines, Koma, Chamba, Duru, Pape et Voko présente une grande similitude avec le rite dowayo". Des bouffons et des "frères d'initiation" y jouent, notamment, un rôle identique ${ }^{32}$. Mais, on ne sait pourquoi, la pétomanie est attachée au seul nom des Koma. Bien que nous n'ayons pas connaissance de récit mentionnant l'occlusion de l'anus dans cette population, l'association qui existe chez les Dowayo entre la circoncision et le pet rituel permet de supposer qu'il existe une association de même nature chez les Koma.

Ces charges scatologiques qui évoquent la circoncision sont, tout au long du cycle funéraire dowayo, adressées au mort par ses "frères d'initiation". Ce sont eux qui, aux côtés des bouffons rituels, le maltraitent ou le ridiculisent tel un circoncis. La participation à une même initiation crée des liens de confraternité qui s'expriment par un échange de prestations rituelles mais la réciprocité ne vaut que pour une poignée d'entre eux. Ceux-là mêmes qui joueront un rôle de décepteur au cours des funérailles. "Ils volent le cadavre, le plongent dans une rivière ou le cachent jusqu'à ce que paiement leur soit fait. A l'enterrement d'un homme pauvre, ils se moquent impitoyablement du mort en apportant en guise de mil, des paniers de cendres ou en dansant avec des peaux de souris comme s'il s'agissait des peaux du bétail abattu en son honneur" (p.71). Aux obsèques d'un homme riche, "ils s'arrêtent tous au milieu de la cérémonie pour aller déféquer ensemble en brousse"(p.74). A leur

\footnotetext{
31 Dans l'un des groupes koma, les Goonu, le terme qui désigne la circoncision, guake, est identique au terme dowayo gaaks. Il diffère cependant dans les autres groupes: dogme pour les Geme et dogmo pour les Gumbe.

32 Toutefois, nous ignorons s'il existe la même catégorie de bouffons chez les Duru.
} 
retour, ils se précipitent sur le cadavre et le bousculent tout en chantant le chant de circoncision.

La description de cette inhumation relevée dans un ouvrage de J.P. Benoit complète ces informations. "Peu à peu la foule se tait, les chants de cinq flûtes montent en choeur. Une voix d'homme entonne un étrange chant syncopé... Cependant qu'on apporte un phallus de bois, un vieillard le tranche en s'appuyant sur le mort. Les joueurs de flûte se lèvent. Le chant, d'abord angoissant, s'apaise et se termine en un cri triomphal, car c'est le chant de la circoncision, celui qu'on fait entendre aux jeunes garçons (juste après l'opération)... En un éclair, deux d'entre eux touchent le sac mortuaire, saisissent l'arc et le carquois sous l'abri et s'enfuient en courant vers la montagne" (1957: 108). On roule alors le cadavre comme un vulgaire ballot jusqu'à sa tombe, située à l'extérieur du village. Avant d'être enseveli verticalement dans la fosse, on détache de la peau la queue du taureau qui est clouée à l'arbre tarko. Cette séparation présage de la décollation à venir. Nous avions remarqué, lors de la préparation du cadavre, que les Koma veillaient à laisser le cou libre de toutes bandelettes pour permettre la décollation ultérieure du crâne. Sachant la corrélation en jeu entre le temps du deuil, l'ancestralisation du défunt et la séparation naturelle du crâne, on pouvait s'interroger sur ces modalités pratiques concernant la coutume dowayo puisque le cadavre est successivement enveloppé dans plusieurs peaux. Or l'auteur relate qu'avant même d'"expédier" le cadavre dans la fosse, "un homme avance un poignard presqu'aussi long qu'un glaive et le plante dans le sac au niveau du cou... Faisant glisser la lame autour du cou, l'homme découpe la première peau de boeuf et en libère un capuchon de cuir qui découvre la seconde peau blanchâtre. Il sectionne de la même manière les trois autres peaux... Chaque capuchon est découpé en lanières qui sont lancées vers les spectateurs... Ils en feront des "médicaments". Enfin la peau de chèvre apparaît; elle laisse voir en partie le visage du mort qu'un ultime coup de poignard met à nu" (Benoit: 110). La tête est donc libérée de toute entrave pour que le défunt accède au monde des ancêtres. Ainsi, plus tard, lors de l'exhumation, lorsque le crâne 

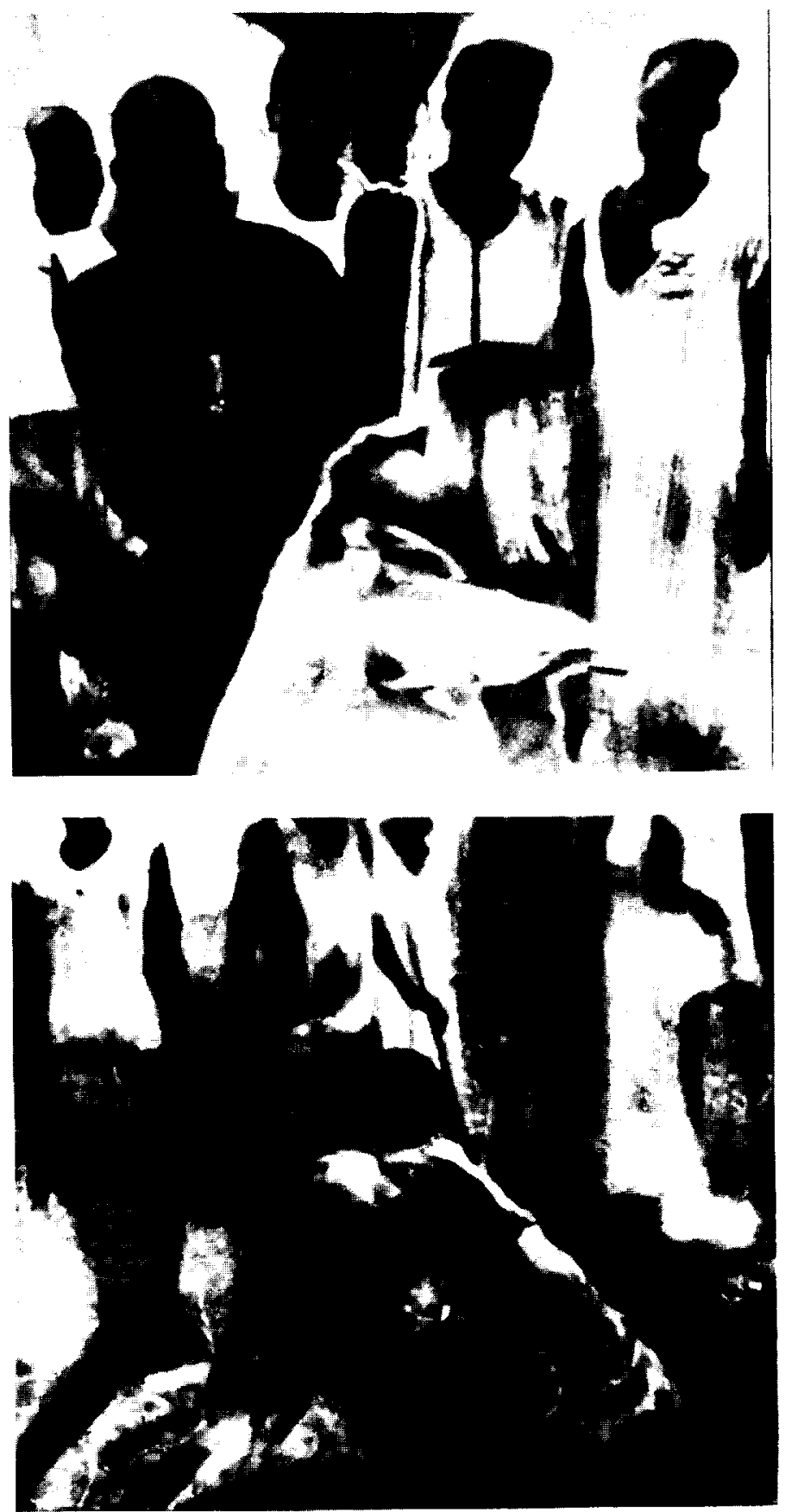

(J.P. Benoit, 1957, p. 79) 
sera "bien sec", "le crochet sera introduit dans l'orbite et le forgeron tirera vers lui en tournant: la tête se décrochera... Il vérifiera si la première vertèbre cervicale n'est pas accrochée au crâne, car cela risquerait de mécontenter l'âme du mort qui reviendrait tourmenter le village" (Benoit:112). La séparation naturelle de la tête est ici encore associée au processus d'ancestralisation.

Au sujet de ce rite, Barley précise que c'est, une fois de plus, l'occasion d'apostropher les femmes dans le registre scatologique. Afin de les éloigner momentanément de la tombe, on leur dit: "Si nous nous courbons pour récupérer le crâne et si nous pétons, le raconterez-vous?" (p. 114 note 14). Ces propos prennent leur véritable sens avec la fête des crânes qui ressemble à "une insulte élaborée à l'égard du mort", alors qu'on réitère sur les boîtes crâniennes le badigeonnage excrémentiel qu'on avait réservé au cadavre. Elles sont, en effet, bombardées de la main gauche avec les déjections des victimes sacrifiées - boucs et béliers - par les organisateurs de la fête et par les clowns qui aspergent, par la même occasion, les assistants parés de leur plus beaux atours. Ce geste rituel est, sans doute, le plus significatif de la cérémonie puisqu'il lui donne son nom: zuuldukyo, qui fait référence à l'action de "jeter" sur les crânes . Lorsqu'il est reproduit en dehors de ce contexte et qu'il consiste à jeter de la saleté, avec la main gauche, au visage de la personne qu'on veut outrager, il équivaut à la pire des insultes et seuls les circoncis ont le droit de la proférer. Le dernier jour de la fête des crânes, un frère d'initiation du mort grimpe sur le toît de son habitation et se frotte l'anus contre le faîte. Il garde cette posture jusqu'à ce qu'on lui offre de la bière. Durant cette parade, les initiés jouent de la flûte, wangbedo, réservée au temps de la circoncision (pp. 102-103).

Ces injures ou ces plaisanteries, tout comme la bouffonnerie des clowns rituels, si elles font explicitement référence à la circoncision, sont aussi la contrepartie des tâches dangereuses que les frères d'initiation accomplissent pour le défunt. Ils deviennent meurtriers, dansent avec les crânes sur la tête (on connaît le danger encouru puisque le transport des crânes est réservé aux vieillards 
qui appartiennent à une catégorie de parents cathartiques, duuse), puis ils libèrent les veuves et les biens personnels du mort, à la fin du deuil.

Les séquences funéraires dowayo ne se déroulent pas selon le même ordre que celles des Koma, si l'on se réfère aux informations fournies par Barley. L'exhumation du crâne, qui intervient "trois semaines après l'enterrement" ${ }^{133}$ et un mois environ avant la levée de deuil, n'est donc pas ici l'acte qui ancestralise le défunt, mais il permet, comme ailleurs, l'examen "phrénologique" qui révèlera la véritable identité du défunt, la présence d'ecchondroses constituant une preuve de sorcellerie (cf. note 14). Si l'exhumation n'est pas l'étape terminale du processus d'ancestralisation, elle est la première opération indispensable à la poursuite du cycle funéraire et sans laquelle le défunt serait, à jamais, voué à l'errance. Le crâne, après avoir été déterré puis nettoyé, est enfermé dans une poterie avant d'être placé dans un arbre. Il y demeure jusqu'à la levée de deuil au cours de laquelle il est transporté dans "l'abri des morts", où se trouvent déjà les objets personnels du défunt: son étui pénien, sa natte, son couteau, sa houe, ses vêtements, son carquois et son arc qui va être coupé à chaque extrémité avec son couteau, traité avec les mêmes éléments que le cadavre, puis "circoncis" (Barley: 89) . Cette coupure, localisée à l'endroit du "nombril", ainsi nommé par les Dowayo, n'est-elle pas plutôt une réitération de la section du cordon ombilical? Quoiqu'il en soit, durant cette opération jugée délicate, tout se passe comme si se jouait la destinée du mort dont l'accomplissement dépend de la fidélité des veuves.

$\mathrm{Au}$ cours de la séquence suivante, tandis que les hommes, portant leur étui pénien, décapitent l'habitation du mort et se ruent en brousse avec le toit en flammes, d'autres évacuent en toute hâte ses effets, saisissent sa calebasse et transportent l'arc et le crâne jusqu'à la "maison des crânes". Le bouffon s'adresse alors aux ancêtres:

33 Ce délai paraît bien court pour que le corps puisse être décomposé. J.C. Casu indique une durée de trois mois (1975: 89) et J.P. Benoit que "le crâne doit être bien sec" (1957: 112). Cependant, il apparaît que les Vere et les Dowayo exhument le crâne après un délai beaucoup plus court que les Koma et les Chamba, qui attendent trois années. 
"Nous avons apporté votre grand fils". La présentation du crâne aux esprits ancestraux, en tant qu'elle apporte la preuve de la mutation du défunt, est l'acte de passage qui permet au mort de rejoindre le monde des ancêtres et, de ce fait, de libérer les deuilleurs. Cependant, tant que le crâne et l'arc ne seront pas installés à l'intérieur de la "maison", l'esprit du mort harcèlera ses descendants pour les contraindre à organiser la cérémonie qui achèvera son ancestralisation.

Une fois le crâne confié aux ancêtres, l'acte de circoncision est mimé. Les hommes entonnent le chant de cette cérémonie et vont coincer le toit de l'habitation du mort entre les branches d'un arbre, tel un crâne qui vient d'être exhumé. Le bouffon, grondant comme un léopard, se jette alors sur le "frère d'initiation", double du défunt, et simule l'opération. Le "circoncis" part s'accroupir dans l'eau de la rivière comme pour rafraîchir sa plaie. Plus tard et publiquement, le mythe d'origine est remis en scène, le bouffon interprétant le rôle de la vieille femme peule.

Les veuves et les orphelins, comme les biens du mort, vont être libérés de son emprise par une aspersion d'eau et de mil germé. Puis les veuves se rendent à un croisement, lieu de passage des ancêtres où s'achève tout rituel. Nous avions vu combien la réussite du rite d'installation de l'arc dépendait de la bonne conduite des épouses. L'une des phases du rituel consistait à attacher un fragment de poterie aux extrémités de l'arc préalablement coupées et trois autres, au niveau du "nombril", où il a été sectionné. Comme pour rappeler les liens qui unissaient les conjoints et qui sont désormais rompus, le bouffon place sur la tête des veuves un même fragment de poterie après l'avoir plongé dans l'huile et l'ocre rouge comme l'arc l'avait été précédemment, puis il le fait tomber en brousse. C'est alors que le frère de circoncision, double du mort, enduit le ventre des veuves d'huile et d'ocre tandis qu'une parente leur oindra le dos. Ce rite de rupture, qui précède la réinsertion des veuves, reproduit celui des circoncis, à leur retour au village. Dèjà, dès l'annonce de la mort, les règles assignées aux veuves rappellent celles auxquelles sont soumis les circoncis. Leur mari est transporté 
dans l'abri des défunts et elles restent assises à l'extérieur, sur des grandes nattes de même facture ${ }^{34}$ que celles des initiants. C'est encore sur ce même genre de natte que sont déposés les crânes lors de la fête qui célébre leur entrée dans la "maison des ancêtres". Chaque soir, la soeur du mort les accompagnaient à la rivière pour qu'elles y prennent un bain, tels les néophytes après l'opération. Elles ont été soumises au même interdit alimentaire que les circoncis durant toute la période du deuil et habillées de feuilles gbungtyo et de végétal silkoh, l'herbe qui blessa le sexe du fils de la vieille femme peule. Si les veuves qui viennent de perdre leur mari sont traitées comme des circoncis, ne sont-elles pas aussi assimilées au défunt, lui-même replacé dans la position d'un initiant? Mais on peut aussi dire des orphelins qui arborent un signe vestimentaire propre à la circoncision qu'ils sont l'objet d'une même identification. Le chapeau rouge de la vieille femme peule qu'ils portent, on en coiffe également le mort. De même, la fille ânée qui intervient dans le rituel comme le substitut de son père, tient encore la même place que le frère d'initiation du défunt et que son arc et son crâne quand, lors de l'enterrement, les femmes originaires du village la transportent sur leurs épaules tout en scandant le chant de guerre. Le confrère du mort est pareillement porté sur les épaules d'un danseur, comme les restes du défunt lors de la cérémonie finale.

La réintégration des veuves correspond à un schéma rituel qui, s'il concorde avec celui des circoncis, s'applique aussi à d'autres participants, selon les circonstances. Ainsi, lorsque le bouffon présente aux veuves un plat, jusque là interdit, de viande fumée et de sésame cuit; ce mets ne leur est pas spécifiquement réservé puisqu'il est de rigueur dans les rites marquant un changement de statut. D'autre part, la tonsure en forme d'étoile qui caractérise les veuves mais surtout les circoncis au moment de leur entrée dans la "maison des crânes", et, à la mort d'une femme, le veuf, les orphelines et ses "soeurs du village", est aussi celle des enfants atteints de zaase.

\footnotetext{
34 Ces nattes sont tressées à partir de graminées ghohgle qui possèdent un "chapeau" rouge appelé la "tête du doré". Au moment des prémices du taro et du gambo, on en fait des tresses qu'on place sur l'autel wagle, comme les crânes.
} 
Les objets quotidiens de la maisonnée sont les agents de cette maladie infantile. Mais on dit encore du couteau de circoncision ou du contact avec les biens du mort qu'ils causent une forme de zaase. On peut dire, pour simplifier, de cette vaste catégorie qu'elle recouvre la notion de souillure. Le traitement que ces cas impliquent reproduit sur la tête des patients les motifs figurant sur les jarres, représentatives des "esprits des morts". Lors de la "décoration" de la jarre, le rite de levée de deuil pour une défunte, on verse dans la poterie, pleine de bière, de la farine de mil germé en appelant la morte par son nom. Le bouillonnement dû à la fermentation est interprété comme la manifestation du principe spirituel de la défunte ${ }^{35}$. L'énumération des défunts a lieu lors de la fête collective des crânes, où les restes des hommes et des femmes sont rassemblés. La jarre féminine est placée sur un foyer, sous un arbre tarko, tandis que l'autre, masculine, est pasée à même le sol, sous un arbre sekoh. (La poterie réservée aux hommes diffère de celle des femmes par un bouchon qui évoque l'anus scellé de la circoncision). Au moment où les duuse versent la bière apportée par l'ensemble des parents, on cite les noms des défunts alors élevés au rang d'ancêtre. De même que le rite d'entrée dans la "maison des crânes" fait des nouveaux circoncis des hommes, lorsque, questionnés sur leur identité, ils divulguent leur nom d'initié pour la première fois, de même, l'ancestralisation des défunts est célébrée par la dation de leur nom. A la fin de la cérémonie, les deux jarres seront entreposées avec les crânes.

Pour finir, les veuves vont toucher le front du bouffon et son épaule avec la patte d'un poulet, trempée dans l'huile et l'ocre. Cette onction, qui intervient à plusieurs reprises dans le cycle funéraire, a des vertus libératrices pour les officiants rituels. Les veuves sont maintenant délivrées de leur veuvage; toutefois, elles ne peuvent pas encore se remarier. Elles doivent attendre la cérémonie des crânes, temps où le défunt est ancestralisé, pour être

\footnotetext{
35 Le mari se met debout, appelle la morte par son nom et dit: "viens, je voudrais t'accompagner aujourd'hui chez ton père" (Casu 1975:95).
} 
héritées par les frères du mort. A cette occasion, elles revivent la même séquence rituelle. Elles passent, une dernière fois, la nuit ensemble sur les nattes ghohgle. Et le lendemain, le bouffon leur présente le plat de viande fumée et de sésame. Comme les circoncis à leur retour au village, elles visitent les habitations de toutes les épouses. La même aspersion sacrificielle avec le sang de la patte d'un poulet a lieu sur le foyer de la première épouse et sur la tête des veuves ainsi que sur celle de la fille de la soeur du mort qui les a vu nues. On leur rase les cheveux en reproduisant les dessins de la jarre. Puis, tels des circoncis, le bouffon leur place entre les cuisses une igname cuite qu'elles doivent manger sans la toucher avec les mains ni la couper avec un couteau. Elles crachent les trois premières bouchées et avalent la quatrième. La consommation de l'igname les rend aptes au mariage. On les pare de bracelets et de colliers (comme la jarre "esprit" ou les patients atteints de zaase) puis on les enduit d'ocre et d'huile avant qu'elles aillent danser avec leur futur époux, les frères du défunt.

Tout au long de son processus d'ancestralisation, le défunt est, nous l'avons vu, placé dans la position d'un circoncis. Le cadavre est habillé comme un néophyte puis associé à une matière végétale excrémentielle ayant valeur initiatique. Le même schéma est reproduit sur l'arc au moment de la levée de deuil. L'arme est coupée à chaque extrémité avant qu'on y attache des morceaux de poterie mais aussi d'écorce yaahutu qui rappelle le traitement du cadavre. L'arc est enfin "circoncis" au niveau du nombril. Sa présentation aux ancêtres, couplée à celle du crâne, donne aussitôt lieu à la mise en scène du mythe d'origine de la circoncision, précédée d'une simulation de l'opération sur le double du défunt. La logique de ce scénario rituel, joué en entier pour la première fois lors du cycle funéraire, montre que le processus d'ancestralisation - en tant qu'il implique l'exhumation du crâne, acte qui parachève la séparation de la tête du reste du corps - est appréhendé comme la reprise de l'acte initiatique de circoncision, séparant l'initiant de son principe féminin. Le fait qu'on ne prélève pas le crâne d'un circoncis mort durant son séjour 
en brousse (Leiris 1934: 75) corrobore cette interprétation (à ses parents, on dira que le léopard l'a dévoré).

Enfin, pour l'intégration du crâne à la maison des ancêtres, un meurtre devait être commis. La mise à mort de la victime était assimilée à celle de la vieille femme peule du mythe. De nos jours, l'organisateur s'écrie à la fin de la cérémonie: "J'ai circoncis ces hommes. Si ce n'était à cause des blancs, j'aurais tué un homme". Le srâne va être, à son tour, "menacé de circoncision" et traité comme le cadavre l'avait été au début des funérailles. L'arc, qui restera associé au crâne, est d'abord enveloppé dans un vêtement funéraire avec une igname provenant de l'abri des morts. Au paquet ainsi confectionné, on attache deux tiges de mil ornées de plumes rouges et blanches. Cet ornement rappelle celui de la jarre à 1'occasion du rite de "décoration" (Barley: 108). L'arc est successivement porté par le bouffon, puis par le frère d'initiation au cours d'un périple qui conduit tous les hommes du village vers la brousse, chantant l'air de circoncision et maniant leurs lances. A leur retour, les hommes déposent le paquet sur le wagle. Puis ils chassent les femmes et les non-initiés avant de célébrer la scène rituelle la plus marquante de la cérémonie. L'organisateur et ses frères se livrent alors à une seconde bataille de déjections animales dont les crânes sont la cible et, pour les asperger de sang, décapitent des poulets que les bouffons tentent de voler. Comme lors de l'exhumation, on nettoie les crânes avec des feuilles de tarko avant de les habiller d'une robe blanche et bleue, d'un chapeau rouge et de peaux de moutons, selon l'habit des circoncis. On "effraie" les crânes ainsi vêtus avec des couteaux en faisant mine de les circoncire. Les duuse les transportent alors sur la tête à travers le village puis en brousse, suivis par les hommes qui chantent l'hymne de guerre. On dépose enfin chaque crâne et son arc sur le wagle. Plus tard, les duuse vont défaire les paquets et, avant de placer les crânes à l'intérieur de la "maison", ils vont les enduire d'huile et d'ocre et les bombarder une nouvelle fois d'excrément animal. 
Au terme de ce travail, noús sommes mieux à même d'appréhender le sens de ce traitement rituel auquel se livrent les populations de la Haute Bénoué. La présentation des faits dowayo autorise une hypothèse sur la signification de la conservation des crânes, hypothèse pertinente pour les sociétés voisines (Duru, Koma, Vere et Chamba) qui présentent à la fois une grande similitude dans le rite de circoncision et dans les modalités du culte des crânes. Nous avons pu établir l'existence d'une corrélation entre le travail du deuil et le processus de décomposition du cadavre: la séparation naturelle de la tête du reste du corps marquant l'accession du défunt au monde des ancêtres inaugurée par l'exhumation du crâne. Cet acte rituel qui confère l'ancestralité doit être appréhendé comme la réitération de l'acte de circoncision. Cette séparation, bien loin d'avoir valeur de perte, opère comme la circoncision une mutation bénéfique puisqu'elle réalise le passage à la condition d'ancêtre.

On peut toutefois s'interroger sur le choix de la circoncision comme modèle. Car enfin, l'acte de circoncire est engagé dans un processus de différenciation des sexes, alors que l'ancestralisation concerne tous les défunts quelque soit leur sexe. Faut-il donc comprendre que seul est retenu de ce procès initiatique la valeur opératoire d'une séparation qui permet un changement de statut? Si l'on compare le traitement réservé aux éléments prélevés du corps, le prépuce et le crâne, on constate qu'ils sont tous deux conservés dans un même lieu, hautement sacralisé. Mais le parallèle s'arrête là puisque le crâne n'a pas la connotation féminine qu'a le prépuce, bien que celui-ci semble emprunter sa sacralité au statut du crâne, en raison d'une homologie structurale. N'est-ce done pas, en fait, l'état d'indifférenciation qu'il s'agit de maitriser? En effet, en supprimant la partie féminine, la circoncision opère le passage à l'état adulte, tandis qu'en séparant la tête du reste du squelette, la décollation du crâne met un terme à l'indétermination du défunt, l'arrache à sa condition d'errant et le fixe définitivement dans l'ancestralité. Les rites mis en scène par les Dowayo illustrent combien "dans l'état du deuil se rejoue quelque chose de l'état d'indifférenciation du mort de même nature que la question de la différence des sexes" (Cartry, 1987, séminaire E.P.H.E.). 


\section{Ouvrages cités}

Adler, A.

1987 "Le royaume moundang de Léré" in Princes et serviteurs du royaume, C. Tardits (éd.), Paris, Société d'Ethnographie, 139-170.

Barley, N.

1983 Symbolic structures. An exploration of the culture of the Dowayos, Cambridge, University Press et Paris, Editions de la Maison des Sciences de l'Homme.

Benoit, J.P.

1957 Kirdi au bord du monde. Un médecin lyonnais au bout du monde, Paris, Julliard.

Casu, J.C.

1975 "La vie sociale des Dowayo. Une ethnie du Nord-Cameroun", Mémoire de maîtrise de l'Ecole Pratique des Hautes Etudes, Ve section, Paris.

Champion, F.

1977 Recherches sur l'organisation sociale des Masa (région de Koumi), Thèse de troisième cycle, Université de Paris V Sorbonne. (Voir aussi Dumas-Champion).

Davies, J.G.

1942-1949 "The Bi Rom. A study of a Nigerian Tribe". Manuscrit déposé à la Bibliothèque du Musée de Jos (Nigeria), $421 \mathrm{p}$.

Dogari, A .

1984 The cultural history of the Koma, Artz Books, Danemark.

Dumas-Champion, F.

1986 "Pierres de vie", in l'Univers du Vivant, 8, 48-61.

1987 "Pouvoir et amertume du fétiche, deux études de cas : les Koma du Cameroun et les Masa du Tchad" in Systèmes de pensée en Afrique noire, VIII, 141-176.

Fardon, R.O.

1980 "The Chamba: a comparative history of tribal politics", $\mathrm{Ph} . \mathrm{D}$. Thesis, London, University College, 2 tomes.

Frobenius, L.

1925 Dichten und Denken im Sudan, V, Atlantis. Trad. Eldridge Mohammadou, Centre de recherches et d'études anthropologiques, Garoua, 1984.

Gauthier, J.G.

1969 Les Fali de Ngoutchoumi, montagnards du Nord-Cameroun, Anthropological Publications, Oosterhout, Pays-Bas. 
Hertz, R.

1928 "Contribution à une étude sur la représentation collective de la mort" in Mélanges de Sociologie Religieuse et Folklore, Paris, Librairie Félix Alcan (1ère ed. 1907).

Lebeuf, J.P.

1961 L'habitation des Fali, Paris, Hachette.

1987 "Le fer, le bois et la fibre" in Ethnologiques, Hommages à Marcel Griaule, Paris, Hermann.

Leiris, M.

1934 "Rites de circoncision namchi" in Journal de la Société des Africanistes, IV, 1, 63-80.

Meek, C.K.

1931a A Sudanese Kingdom, London, Kegan Paul, Trench, Trubner and $\mathrm{C}^{\circ}$.

1931b Tribal studies in Northern Nigeria, 2 vol., London, Kegan Paul, Trench, Trubner and $\mathrm{C}^{\circ}$.

Mohammadou, E.

1978 Les royaumes foulbé du plateau de l'Adamaoua au XIXe siècle, Tokyo, I.L.C.A.A. (Institute for the Study of Langruages and Cultures of Asia and Africa).

Moore, S.F.

1976 "The secret of the men: a fiction of Chagga initiation and its relation to the logic of Chagga symbolism", Africa, XLVI, 4, 357-369.

Podlewski, A.

1971 La dynamique des principales populations du Nord-Cameroun, Cahier ORSTOM., vol. VIII, $\mathrm{n}^{\mathbf{0}}$ spécial.

Pradelles de Latour Dejean, C.H.

1979 "Les sacrifices faits aux ancêtres chez les Bangwa", in Systèmes de pensée en Afrique noire, 4, 131-138.

1986 Iue champ du langage dans une chefferie bamiléké, Thèse d'Etat, Ecole des Hautes Etudes en Sciences Sociales.

Tardits $\mathrm{C}$.

1980 Le royaume bamoun, Paris, Armand Colin.

Yamaguchi M.

1974 "La royauté et le symbolisme dualiste chez les Jukun du Nigeria", Journal of Asian and African Studies, 8, Tokyo, $1-29$. 